\title{
Keratinocyte growth factor-induced hyperplasia of rat alveolar type II cells in vivo is resolved by differentiation into type I cells and by apoptosis
}

\author{
H. Fehrenbach*, M. Kasper**, T. Tschernig***, T. Pan ${ }^{+}$, D. Schuh*, \\ J.M. Shannon ${ }^{+}$, M. Müller*, R.J. Mason ${ }^{+}$
}

\begin{abstract}
Keratinocyte growth factor-induced hyperplasia of rat alveolar type II cells in vivo is resolved by differentiation into type I cells and by apoptosis. H. Fehrenbach, M. Kasper, T. Tschernig, T. Pan, D. Schuh, J.M. Shannon, M. Müller, R.J. Mason. (C)ERS Journals Ltd 1999. ABSTRACT: Keratinocyte growth factor (KGF) is a potent mitogen of alveolar epithelial type II cells (AEII). AEII hyperplasia is resolved within several days following intratracheal instillation of KGF by unknown mechanism(s).

AEII hyperplasia was induced in rat lungs by intrabronchial instillation of $5 \mathrm{mg}$ recombinant human (rh)KGF $\mathrm{kg}_{\text {body }}$ weight $^{-1}$ or an equivalent amount of diluent. Epithelial architecture, cell proliferation, transformation of AEII into type I cells (AEI) and apoptosis were investigated by means of immunohistochemistry, stereology, double immunofluorescence microscopy, electron microscopy and the terminal deoxynucleotidyl transferase-mediated deoxyuridine triphosphate nick end-labelling (TUNEL) technique in lungs fixed 1, 2, 3 and 7 days after treatment.

After 1 day of rhKGF instillation, an increase was observed in the nuclear antigen Ki-67, a proliferation marker detected by the antibody MIB-5-expressing surfactant protein (SP)-B, -C, -D-positive AEII. The incidence of mitosis was increased by day 2, resulting in AEII micropapillae with intense basolateral expression of the exon 6 containing isoform (v6) of CD446 (CD44v6), a marker for AEII. By day 3, monolayers of AEII exhibiting lateral CD44v6 covered 45\% of the alveolar surface. After 7 days, there were numerous intermediate AEII/AEI cells characterized by a flat elongated shape, staining for SP-D, apical appearance of AEI marker Lycopersicon esculentum lectin and lateral staining for AEII marker CD44v6. Increased numbers of TUNELpositive epithelial cells were seen at days $2-7$.

In conclusion, restoration of normal alveolar epithelium after instillation of recombinant human keratinocyte growth factor is accomplished by terminal differentiation and apoptosis of hyperplastic alveolar epithelial type II cells in vivo.

Eur Respir J 1999; 14: 534-544ol.
\end{abstract}

*Institute of Pathology and **Institute of Anatomy, University Clinics "Carl Gustav Carus", Technical University of Dresden, Germany. ***Functional and Applied Anatomy, Hannover Medical School, Hannover, Germany. ${ }^{+}$National Jewish Medical and Research Center, Denver, CO, USA.

Correspondence: H. Fehrenbach, Institute of Pathology, University Clinics "Carl Gustav Carus", TU Dresden, Fetscherstr. 74, D-01307 Dresden, Germany. Fax: 49 3514584328

Keywords: Alveolar epithelium apoptosis

differentiation

hyperplasia

keratinocyte growth factor type II cell

Received: February 221999

Accepted after revision July 21999

This work was supported by a grant from the National Institutes of Health, USA (HL56556), and by the Bundesministerium für Bildung und Forschung, Germany (01ZZ5904).
Keratinocyte growth factor (KGF) is a heparin-binding stroma-derived member of the fibroblast growth factor family which selectively or at least predominantly stimulates the proliferation of epithelial cells [1-4]. In the lung, $\mathrm{KGF}$ has been demonstrated to act as a potent mitogen of alveolar epithelial type II cells (AEII) in vitro [5-7] and in vivo $[8,9]$. Studies of the kinetics of the incorporation of 5-bromo-2'-deoxyuridine (BrdU) into alveolar cells showed that hyperplasia of AEII peaks at $\sim 2-3$ days after treatment with recombinant human (rh)KGF given via the bronchial [8] or vascular route of administration [9]. At the time of AEII hyperplasia, the lungs are protected against various forms of injury including acid instillation [10], $\alpha$-naphthylthiourea [11], hyperoxia [9], bleomycin $[9,12-14]$, and $\gamma$-irradiation [12]. In these experiments, the animals were pretreated with rhKGF before the injury, and attempts at post-treatment have not been successful to date [13]. Consequently, the prevention of cell loss has been proposed to be an important mechanism by which rhKGF realizes this high protective potential [14].

Although it has been reported that, 1 week after intratracheal treatment with rhKGF, the lungs' parenchymal architecture was indistinguishable from that of control lungs [8], nothing is known about the mechanism(s) by which the proliferative effect of rhKGF is resolved. Therefore, this study sought to investigate the mechanism(s) of restoration of a normal alveolar epithelium following rhKGF-induced AEII hyperplasia. In particular, the hypothesis was tested that resolution of hyperplasia is achieved by differentiation of AEII into type I cells (AEI) and/or by apoptosis.

\section{Material and methods}

\section{Study design}

Fisher 344 rats received a single intratracheal instillation of $5 \mathrm{mg} \cdot \mathrm{kg}$ body weight ${ }^{-1} \mathrm{rhKGF}$ or an equivalent volume of the buffer used for dilution of rhKGF. To determine the time course of the action of rhKGF on the alveolar epithelium, two animals were sacrificed 1,2, 3 and 7 days after treatment. In addition, the lungs of two uninstilled rats were also examined. The lungs were removed, fixed in $4 \%$ paraformaldehyde and embedded in paraffin. To examine alterations in the parenchymal architecture, cell proliferation, transformation of AEII into AEI, and the 
occurrence of apoptosis, the lungs were investigated by means of immunohistochemistry and double immunofluorescence microscopy using well-established antibodies and lectins [15-22], transmission electron microscopy [23], and the terminal deoxyribonucleotidyl transferase (TdT)mediated deoxyuridine triphosphate (dUTP) nick end-labelling (TUNEL) technique for the assessment of apoptosis [24]. In order to quantify the effects of rhKGF on the alveolar epithelium, stereology was used in conjunction with immunohistochemistry to obtain an estimate of the fraction of the alveolar wall surface covered by AEII and AEII/AEI intermediates at each time point [25].

\section{Methods}

Intrabronchial instillation of recombinant human keratinocyte growth factor. rhKGF prepared at Amgen Inc. (Thousand Oaks, CA, USA) was produced in Escherichia coli, purified to homogeneity by conventional methods, found free of endotoxin and assayed for biological activity using the Balb/MK keratinocyte cell line [26]. Following previous studies $[8,13]$, anaesthetized animals were placed in the supine position, and intubated orally with a 16-gauge intravascular Teflon catheter (QuikCath, Baxter Deerfield, IL, USA) under direct visualization. The intratracheal catheter was curved slightly and inserted to point toward the left lung, and, through the intratracheal catheter, a fine curved catheter was inserted through which the rhKGF or diluent was instilled into the left lung [10, 13]. In each animal, rhKGF was administrated as a single bolus at a dose of $5 \mathrm{mg} \cdot \mathrm{kg}$ body weight ${ }^{-1}$ diluted in 300 $\mu \mathrm{L}$ buffer vehicle followed by a single inflation of $0.5 \mathrm{~mL}$ air. Vehicle control animals received $300 \mu \mathrm{L}$ diluent followed by a single inflation of $0.5 \mathrm{~mL}$ air.

Fixation and tissue processing. For immunohistochemistry and immunofluorescence microscopy, the lungs were perfused with phosphate-buffered saline (PBS, $\mathrm{pH}$ 7.4) via the right ventricle immediately after sacrifice, and subsequently instilled via the trachea with 4\% paraformaldehyde in PBS. The left lung was cut into equidistant slices, which were embedded in paraffin.

For electron microscopy, lungs were instilled with a mixture of $4 \%$ paraformaldehyde and $0.5 \%$ glutaraldehyde in $0.1 \mathrm{M}, \mathrm{pH} 7.3$ cacodylate buffer at a hydrostatic pressure of $20 \mathrm{cmH}_{2} \mathrm{O}$. After ligation of the trachea, lungs were placed into fixative overnight $\left(8^{\circ} \mathrm{C}\right)$. The next day, tissue blocks were collected from the left lung according to a systematic random sampling scheme, post-fixed in $1 \%$ osmium tetroxide in $0.1 \mathrm{M}$ cacodylate buffer, stained en bloc with half-saturated aqueous uranyl acetate, as described previously [23], dehydrated through a graded series of alcohol and embedded in Vestopal (Serva, Hamburg, Germany).

Immunohistochemistry. Dewaxed paraffin sections were stained by means of established immunohistochemical procedures, described in detail in a number of previous studies [16-20,27]. In brief, sections were incubated with hydrogen peroxide to eliminate endogenous peroxidase, and unspecific staining was blocked using 50\% foetal calf serum in PBS prior to incubation with the primary antibodies or lectins used at the appropriate dilution (see table 1). After washing in PBS for $10 \mathrm{~min}$, antibodies were detected by incubation for $60 \mathrm{~min}$ with peroxidase-coupled goat antirabbit immunoglobulin G (HRP77; H. Grossmann, Dresden, Germany) at a 1:400 dilution in PBS containing $50 \%$ foetal calf serum followed by $0.6 \%$ diaminobenzidine in PBS for $8 \mathrm{~min}$. Alternatively, the avidin/biotin/peroxidase complex (ABC) technique (Vectastain Elite kit; Vector Laboratories, Burlingame, CA, USA) was used where appropriate. After rinsing in PBS, brief counter-staining with haematoxylin was performed.

Double immunofluorescence microscopy. Dewaxed paraffin sections were stained, as previously described in detail $[16,19]$. Briefly, sections were washed in PBS, incubated with primary antibody or lectin diluted in PBS, labelled with 4,6-dichlorotriazinyl-aminofluorescein and incubated with the second antibody or lectin, which was finally labelled with Texas red. Sections were mounted in glycerol:PBS (9:1) containing 2.5\% 1,4-diazobicyclo[2.2.2]-octane (DABCO; Janssen, Beerse, Belgium) to reduce fading of fluorescent dyes. Sections were examined using an Olympus BH-2 microscope equipped with a reflected light fluorescence device (Olympus, Tokyo, Japan).

Electron microscopy. Ultrathin sections were collected on 200 -mesh nickel grids coated with $3 \%$ collodion. Counter-staining was performed with $2 \%$ uranyl acetate and Reynold's lead citrate following standard procedures. A Zeiss EM 900 (Zeiss, Oberhochen, Germany) transmission electron microscope, operated at $80 \mathrm{kV}$, was used for ultrastructural analysis.

Assessment of apoptosis. The TUNEL method, an established technique for evaluating the presence of apoptotic cells [24], was used to demonstrate apoptosis in dewaxed paraffin sections by means of two different kits (APAAP in situ cell death detection kit; BoehringerMannheim; ApopTag peroxidase in situ apoptosis detection S7100-kit, Oncor-Appligene). Both kits are based on the TdT catalysed polymerisation of nucleotides (dUTP) to free $3^{\prime}-\mathrm{OH}$ deoxyribonucleic acid (DNA) ends characteristic of apoptosis-associated DNA strand breaks. To exclude any effect of the secondary detection system, an alkaline phosphatase/antialkaline phosphatase system was used in one kit and an anti-digoxigenin/peroxidase system in the other kit. As a control, consecutive sections were treated identically with only TdT being omitted in the primary incubation step. With both kits, equivalent results were obtained.

Stereology. In order to quantify the changes in alveolar wall architecture with time after rhKGF treatment, one paraffin section of each lung containing two-three slices was stained for surfactant protein (SP)D, and hyperplasia of AEII as well as occurrence of AEII/AEI intermediates were analysed by means of standard stereology [25]. Starting from a random position outside the section, each section was completely scanned to include all slices by moving the microscope stage a constant distance along the $\mathrm{x} / \mathrm{y}$ axes. At each position, a cycloidal test grid was projected into the virtual section image of the microscope, and the architecture of the parenchyma was evaluated as follows. To estimate the percentage of 
Table 1. - Antibodies, lectins and kits used to investigate parenchymal alterations in rat lungs instilled with recombinant human keratinocyte growth factor

\begin{tabular}{|c|c|c|c|c|c|c|}
\hline Marker & Main specificity & Type of marker & Origin & Dilution & Detection system & Ref. \\
\hline $\begin{array}{l}\text { Lycopersicon } \\
\text { esculentum lectin }\end{array}$ & $\begin{array}{l}\text { AEI, ciliated airway } \\
\text { epithelium }\end{array}$ & $\begin{array}{l}\text { Lectin specific for } \\
N \text {-acetylglu- } \\
\text { cosamine }\end{array}$ & Vector Laboratories & $1: 200$ & $\mathrm{ABC}$ & {$[28]$} \\
\hline Caveolin & AEI, endothelium & $\begin{array}{l}\text { Rabbit antihuman } \\
\text { polyclonal anti- } \\
\text { body }\end{array}$ & $\begin{array}{l}\text { Transduction } \\
\text { Laboratories } \\
\text { (Dianova) }\end{array}$ & $1: 20$ & $\begin{array}{l}\text { Peroxidase-coupled } \\
\text { Goat antirabbit IgG }\end{array}$ & {$[20]$} \\
\hline SP-B & AEII, Clara cells & $\begin{array}{l}\text { Rabbit antihuman } \\
\text { polyclonal antibody }\end{array}$ & Chemicon & $1: 25$ & Antirabbit kit & \\
\hline SP-C & AEII & $\begin{array}{l}\text { Rabbit antihuman } \\
\text { polyclonal anti- } \\
\text { body }\end{array}$ & J.A. Whitsett & $1: 50$ & $\begin{array}{l}\text { Peroxidase-coupled } \\
\text { Goat antirabbit IgG }\end{array}$ & {$[15]$} \\
\hline SP-D & $\begin{array}{l}\text { AEII, alveolar } \\
\text { macrophages, } \\
\text { Clara cells }\end{array}$ & $\begin{array}{l}\text { Mouse antirat } \\
\text { monoclonal } \\
\text { antibody }\end{array}$ & S. Albrecht & Undiluted & $\begin{array}{l}\text { Peroxidase-coupled } \\
\text { Goat antirabbit IgG }\end{array}$ & {$[17]$} \\
\hline Cytokeratin 18 & AEIII & $\begin{array}{l}\text { Mouse antihuman } \\
\text { monoclonal anti- } \\
\text { body (clone Ks18.04) }\end{array}$ & Progen & $1: 100$ & $\mathrm{ABC}$ & {$[27]$} \\
\hline CD44v6 & $\begin{array}{c}\text { AEII, airway } \\
\text { epithelium }\end{array}$ & $\begin{array}{l}\text { Mouse antirat mono- } \\
\text { clonal antibody } \\
\text { (clone } 1.1 \text { AMSL) }\end{array}$ & U. Günthert & Undiluted & $\mathrm{ABC}$ & [19] \\
\hline $\mathrm{Ki}-67$ & Proliferating cells & $\begin{array}{l}\text { Mouse antirat mono- } \\
\text { clonal antibody } \\
\text { (clone MIB-5) }\end{array}$ & Dianova & $1: 10$ & $\mathrm{ABC}$ & {$[21]$} \\
\hline TUNEL & Apoptotic cells & TdT, dUTP & $\begin{array}{l}\text { Boehringer-Mann- } \\
\text { heim, (Oncor- } \\
\text { Appligene) }\end{array}$ & - & $\begin{array}{l}\text { APAAP } \\
\text { ABC }\end{array}$ & [29] \\
\hline
\end{tabular}

SP: surfactant protein; CD44v6: the exon 6 containing isoform of CD44; Ki-67: a nuclear antigen; TUNEL: terminal deoxynucleotidyl transferase (TdT)-mediated deoxyuridine triphosphate (dUTP) nick end-labelling technique; AEI: alveolar epithelial type I cells; AEII: type II cells; AEIII: type III cells; ABC: avidin/biotin/peroxidasse complex; IgG: immunoglobulin G; APAAP: alkaline phosphatase/ antialkaline phosphatase; Ref: reference; Vector Laboratories: Burlinghame, CA, USA; Transduction Laboratories: San Diego, CA, USA; Dianova: Hamburg, Germany; Chemicon: Temecula, CA, USA; J.A. Whitsett: Cincinatti, OH, USA; S. Albrecht: Dresden, Germany; Progen: Heidelburg, Germany; U. Günthert: Basle, Switzerland; Boehringer-Mannheim: Mannheim, Germany; OncorAppligene: Gaitherburg, MD, USA.

alveolar wall surface covered by AEI, AEII and AEII/ AEI intermediates, all intersections of the test lines with the alveolar wall basal lamina (IEpi), the SP-D-stained cuboid AEII (IPII) and the lentil-shaped flat elongated SP-D-stained AEII/AEI intermediates (IPII/I) were counted, respectively. For each individual lung, the sums of all the intersections, i.e. $\Sigma$ IEpi, $\Sigma$ IPII and $\Sigma$ IPII/I were determined, and the fraction of the alveolar wall surface which was covered by the different cell types was calculated according to the following formulae: 1) surface fraction of alveolar wall covered by type II cells (Ss $($ PII,Epi) $)=\Sigma$ IPII $/ \Sigma$ IEpi; 2) surface fraction of alveolar wall covered by AEI/AEII intermediates (Ss $(\mathrm{PII} / \mathrm{I}, \mathrm{Epi}))=$ $\Sigma$ IPII/I/ $/$ IEpi; and 3 ) surface fraction of alveolar wall covered by AEI $($ Ss $($ PI,Epi $))=(\Sigma$ IEpi $-\Sigma$ IPII $-\Sigma$ IPII $/$ I) $/ \Sigma$ IEpi.

Statistics. Analysis of variance (ANOVA) and partial correlation analysis were used to determine the level of significance of the influence of the treatment with rhKGF versus saline on the stereologically estimated increase in AEII and of AEII/AEI intermediates, respectively. A pvalue of 0.05 was considered significant.

\section{Results}

In accordance with earlier studies [8, 13], intrabronchial instillation of $5 \mathrm{mg}$ rhKGF. $\mathrm{kg}$ body weight ${ }^{-1}$ into rat lungs resulted in prominent hyperplasia of AEII (fig. 1).
The time course of the alveolar epithelial changes exhibited the characteristic micropapillary epithelial cell hyperplasia or "knobby proliferation" at day 2 after rhKGF instillation, "diffuse proliferation" in the form of monolayers of cuboidal epithelial cells lining alveolar septa at day 3 and an almost normal histological appearance at day 7 , as described previously [8]. These alterations in the alveolar epithelium could also be quantitatively demonstrated by means of stereology in conjunction with immunohistochemical analysis of SP-D (fig. 2). While in the normal rat lung only $\sim 4 \%$ of the alveolar surface is covered by AEII [30], these cells covered $\sim 25 \%$ of the alveolar surface at day 2 and $\sim 45 \%$ at day 3 after instillation of rhKGF. At days 1 and 7, the surface fraction of alveolar wall covered by AEII was equivalent (5-10\%) to that of untreated and saline-treated lungs (5-9\%). The increase in AEII coverage of the alveolar wall from day 0-3 after rhKGF treatment versus that of diluent was statistically significant at $\mathrm{p}<0.05$.

AEII hyperplasia was not observed until day 2 after instillation of rhKGF, when mitotic figures were seen frequently (fig. 1b). However, staining for the nuclear antigen and proliferation marker Ki-67 was remarkably increased as early as day 1 following rhKGF treatment, peaked at days 2 and 3, and was again comparable to untreated lungs and diluent controls at day 7 (fig. 1e-h). Double immunofluorescence microscopy for Ki-67 and SP-C revealed that almost all cells exhibiting nuclear 

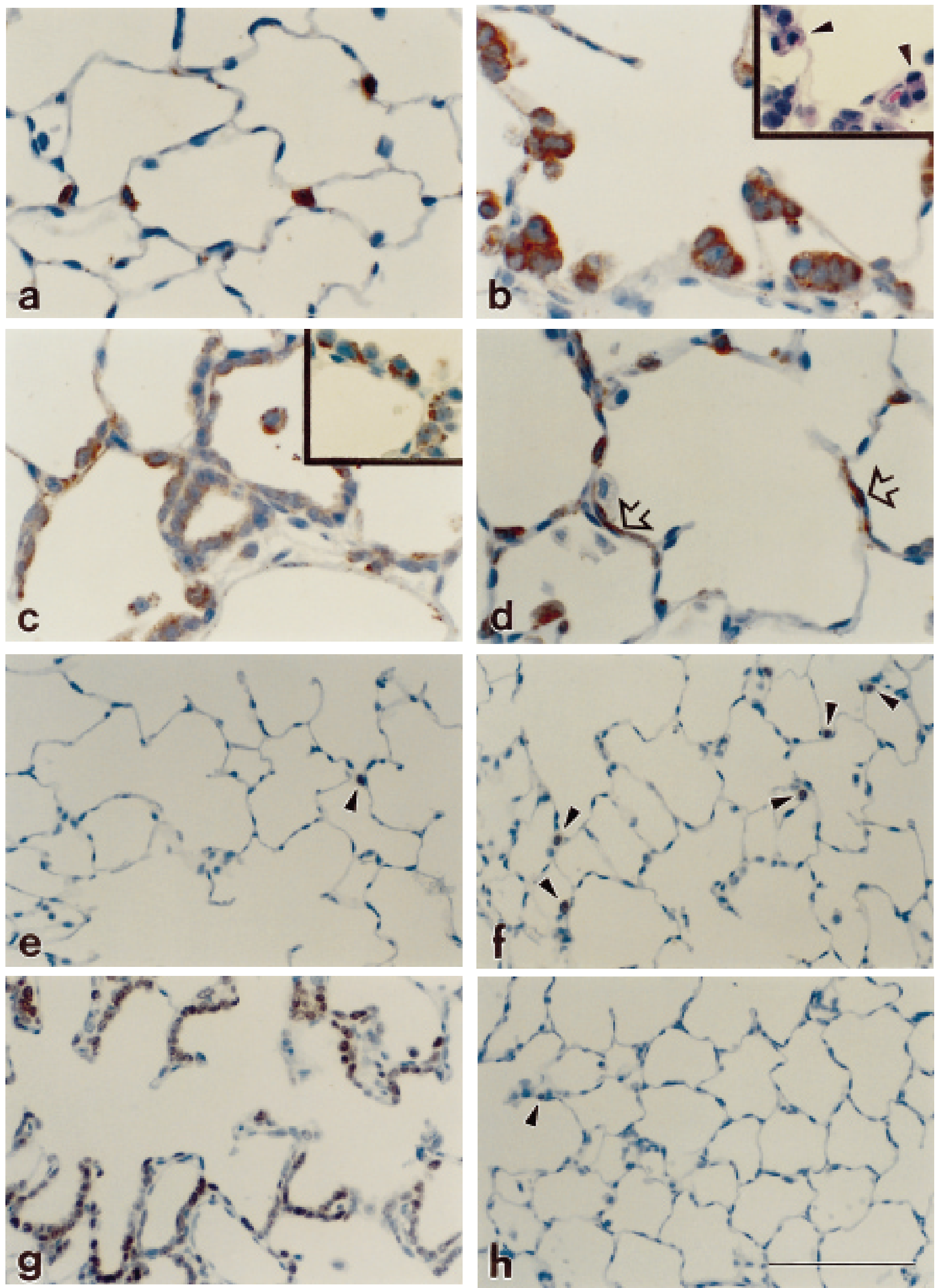

Fig. 1. - Immunohistochemistry of rat lung parenchyma stained for: a-d) surfactant protein D (SP-D); and e-h) proliferation marker Ki-67. Control lungs, intrabronchially instilled with diluent, exhibit: a) single alveolar epithelial type II cells (AEII) located in corners of alveoli; and e) exceptional nuclear staining for Ki-67 (arrowhead) after: e) $24 \mathrm{~h}$; and a) 3 days. Lungs intrabronchially instilled with recombinant human keratinocyte growth factor exhibited; f) increased incidence of Ki-67-staining after $24 \mathrm{~h}$; b) typical micropapillary AEII hyperplasia at day 2 associated with a high incidence of mitotic figures (see inset, haematoxylin/eosin staining); c, g) cuboid alveolar epithelial monolayers at day 3 (inset in c, surfactant protein B staining); and d, h) almost normal architecture at day 7 with numerous flat elongated SP-D-staining alveolar epithelial cells (open arrows). (Internal scale bars a-d=50 $\mu \mathrm{m}$; e-h=100 $\mu \mathrm{m}$. 


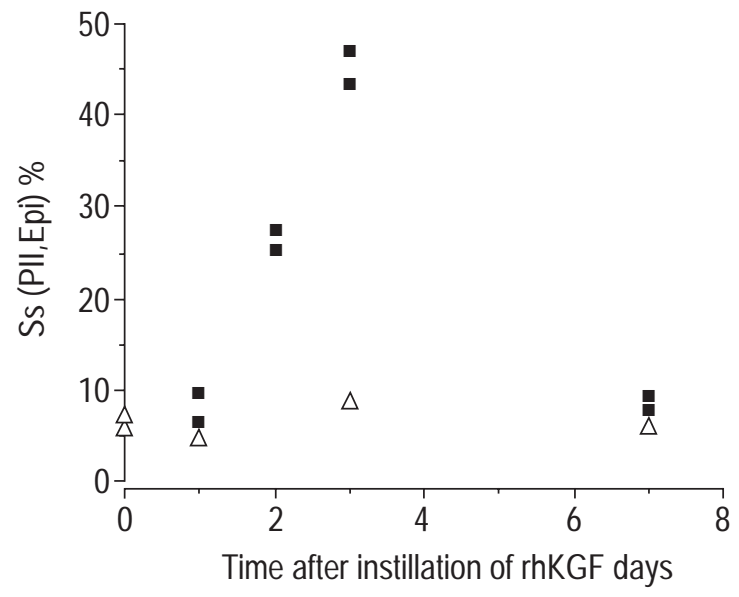

Fig. 2. - Alveolar epithelial type II cell (AEII) hyperplasia following intrabronchial instillation of recombinant human keratinocyte growth factor (rhKGF) into rat lungs estimated by means of stereology based on morphology and surfactant protein D immunohistochemistry. The increase in AEII coverage of the alveolar wall (Ss (PII,Epi)) day 0-3 following treatment with rhKGF $(\boldsymbol{\square})$ versus diluent $(\triangle)$ was statistically significant at a level of $p=0.030$ and $p=0.029$, tested by analysis of variance and partial correlation, respectively.

staining for this proliferation marker were AEII (fig. 3a and d).

Hyperplasia of AEII was accompanied by a prominent increase in the staining intensity for CD44v6 (fig. 4), which selectively labels the basolateral cell membrane of AEII. In addition, rhKGF instillation resulted in a shift in the distribution pattern from a basolateral to a predominantly lateral cell membrane-staining at day 3 (fig. 4c), when the alveolar wall had acquired the appearance of a cuboid epithelial monolayer. The progressive expansion of AEII over the alveolar surface during the first 3 days following instillation of rhKGF was accomplished at the expense of AEI. This could be demonstrated by means of immunohistochemical staining for caveolin (fig. $3 \mathrm{~b}$ and e), intercellular adhesion molecule 1 (not shown) and Lycopersicon esculentum lectin (LEL) (fig. 3c and f) which have previously been shown to be present on AEI but not on AEII $[16,20,28]$. At day 7 after rhKGF treatment, AEI had recovered the alveolar surface; this was associated with an increased cellularity of the alveolar septa (fig. 1d and h).

However, by day 3 after instillation of rhKGF, double immunofluorescence microscopy revealed that numerous SP-D-staining AEII also exhibited faint apical staining for the AEI marker LEL (fig. 3c and f). Using electron microscopy AEII monolayers being covered by AEI or vice versa were never observed (fig. 5). In contrast to the AEII of control lungs, hyperplastic AEII of rhKGF-instilled lungs exhibited apical staining for cytokeratin 18 (fig. 4 $\mathrm{e}-\mathrm{h})$. Further, an increased number of lentil-shaped flat elongated albeit SP-D-staining AEII/AEI cell intermediates was noted at day 7 after rhKGF treatment. In places, these intermediate cells were seen to exhibit CD44v6 staining of their lateral but not of their basal cell membrane (fig. 4d). The fractions of the total alveolar wall surface covered by these AEII/AEI intermediates were stereologically shown to increase day $2-7$, reaching an alveolar surface fraction of $\sim 6 \%$ at day 7 as compared to only $0.1-0.8 \%$ in control lungs (fig. 6). The increase in
AEII/ AEI coverage of the alveolar wall day 0-7 after rhKGF versus diluent treatment was statistically significant at $\mathrm{p}<0.05$.

Only single apoptotic cells were seen in sections of untreated and saline-treated lungs stained by means of the TUNEL method. In lungs that had received a single intrabronchial instillation of rhKGF, however, TUNEL-positive alveolar epithelial cells were regularly observed by day 2 after treatment. The increased incidence of apoptotic cells, which almost completely consisted of alveolar epithelial cells, was even more prominent at day 7 (fig. 7).

\section{Discussion}

The present study of alveolar epithelial remodelling following a single intratracheal instillation of rhKGF into rat lungs, which is based on immunohistochemistry, double immunofluorescence microscopy, electron microscopy, stereology and the TUNEL technique, revealed that restoration of a normal parenchymal architecture was achieved in vivo by both differentiation of hyperplastic AEII into AEI and apoptosis. The time course of the major events seen in AEII after instillation of rhKGF is schematically summarized in figure 8 .

The pattern of alveolar epithelial remodelling following rhKGF instillation observed in the present experiments was fundamentally identical to the histological changes reported from previous in vivo studies $[8,9]$. Lungs instilled with rhKGF exhibited the characteristic histological appearance of a micropapillary proliferation of AEII after 2 days, monolayers of cuboidal AEII lining the alveolar septa after 3 days and a near to normal histology after 7 days [8]. This sequence of remodelling and restoration of alveolar septa was quantified by means of stereologic estimates, i.e. by calculation of Ss (PII,Epi). In contrast to the commonly used counting of cell profiles per field of view, this stereological approach provides a basis for the comparison of data obtained by different laboratories in completely different studies, as shown by the equivalence of the Ss (PII,Epi) of 5-7\% estimated in the present immunohistochemical study and the Ss (PII, Epi) of 4\% estimated by electron microscopy for normal rat lungs [30].

Whereas, in the normal lung, only $0.3-6.5 \%$ of AEII are in distinct phases of the cell cycle, as reviewed recently [31], almost all AEII had entered the cell cycle by day 1 after rhKGF instillation, as can be inferred from the staining pattern for the nuclear antigen $\mathrm{Ki}-67$. This is in accordance with studies on the incorporation of BrdU by alveolar epithelial cells [8, 9]. However, the Ki-67 proliferation marker does not stain only a subpopulation of proliferating cells, as is achieved, for example, by staining for incorporated BrdU (cells in the deoxyribonucleic acid synthesis (S) phase only) or for proliferating cell nuclear antigen (cells of late interphase $(G)_{1}, S$, and early $\mathrm{G}_{2}$-phase), but is found in all phases (including mitosis (M)) except early $\mathrm{G}_{1}$ [32]. Thus, Ki-67 staining appears to be a better means of estimating the proliferative activity than any of the other phase-specific markers [31]. The formation of micropapillae, which comprise four or more AEII at day 2, indicates that the cells do not enter the $G_{0}$ phase after the $\mathrm{M}$ phase, but run through one or more 

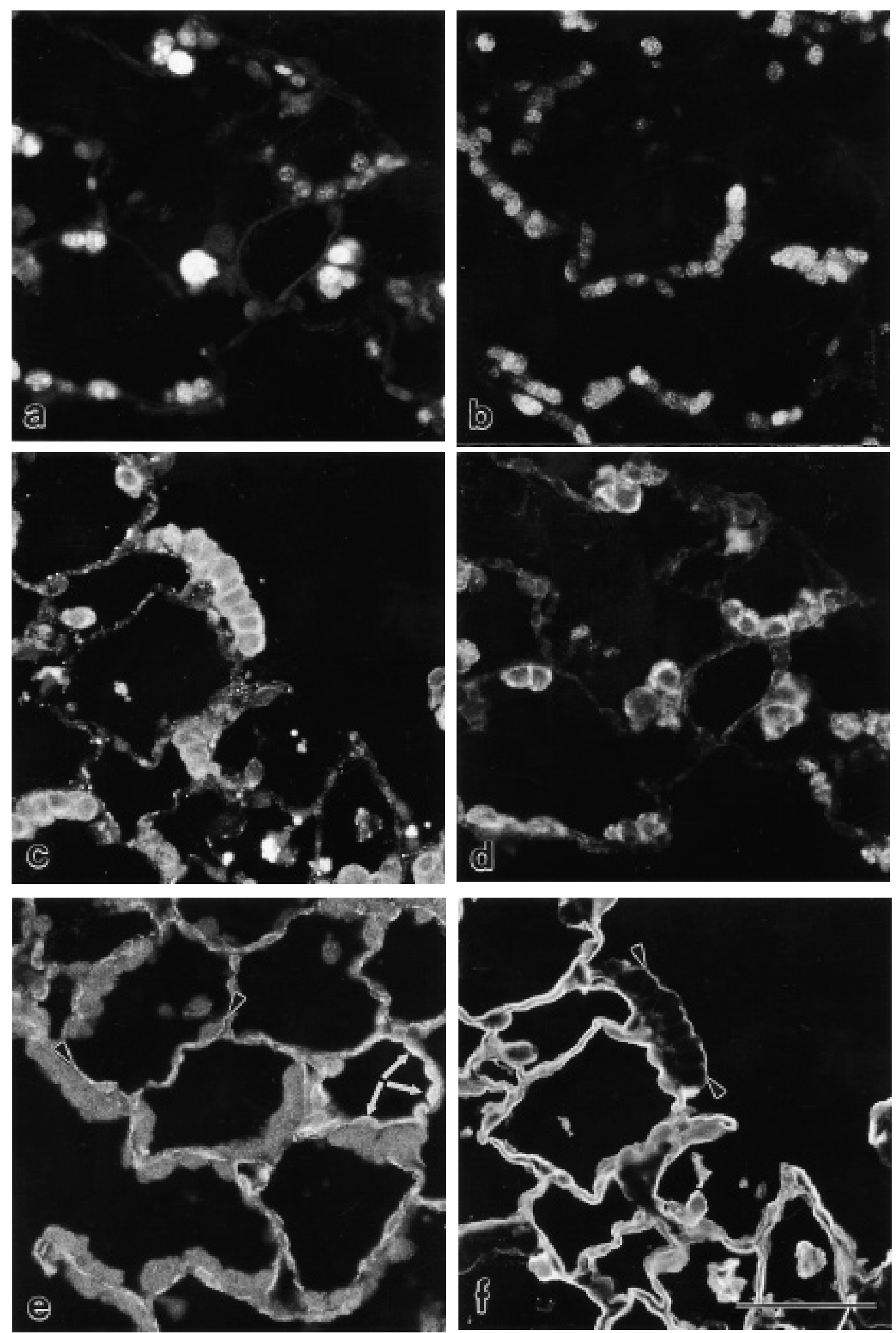

Fig. 3. - Double immunofluorescence staining (DIF) of lungs: a, c, d, f) 2 days; and b, e) 3 days after intrabronchial instillation of recombinant human keratinocyte growth factor. DIF for:a)Ki-67; andd) surfactant protein(SP)C revealed that almostall alveolar epithelial type II cells(AEII)were in the cell cycle, and that almost all cells staining for Ki-67 were AEII. DIF for: b) Ki67; and e) caveolin showing that proliferating alveolar epithelial cells do not grow over but supplant thin caveolin-positive type I cell (AEI) leaflets (arrowheads); white arrows mark caveolin-positive venular endothelium. DIF for: c) SP-D; and f) Lycopersicum esculentum lectin (LEL) revealed that proliferating AEII exhibit apical staining for the AEI marker LEL. (Internal scale bar=50 $\mu \mathrm{m}$.) 

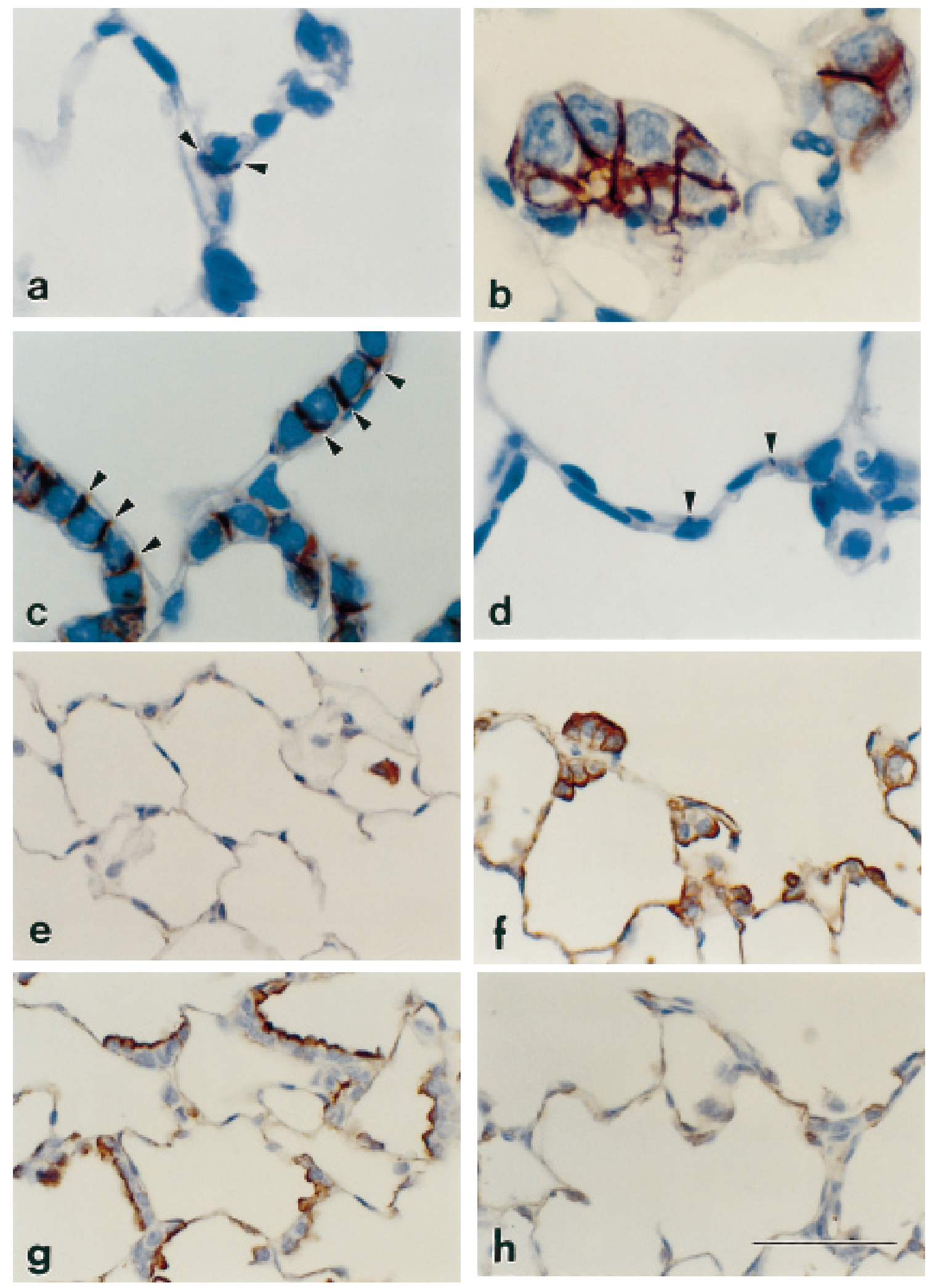

Fig. 4. - Immunohistochemistry of rat lung parenchyma stained for a-d) CD44v6; and e-h) cytokeratin 18 (CK 18) of: a, e) uninstilled control lungs; and $\mathrm{b}-\mathrm{d}, \mathrm{f}-\mathrm{h}$ ) lungs which were fixed: b, f) 2 days; c, g) 3 days; and d, h) 7 days after intrabronchial instillation of recombinant human keratinocyte growth factor (rhKGF). Control lungs exhibit weak basolateral membrane staining of alveolar epithelial type II cells for CD44v6 (arrowheads), and only trace amounts of staining for CK 18. Lungs treated with rhKGF exhibit: $\mathrm{b}$, f) considerably increased staining intensities for both CD44v6 and CK 18 at day $2 ; \mathrm{c}, \mathrm{g}$ ) predominant lateral membrane staining for CD44v6 and intense apical staining for CK 18 at day 3 ; and d, h) almost normal appearance at day 7. In d) a flat elongated alveolar epithelial cell with discrete lateral membrane staining (arrowheads) is shown. (Internal scale bar=a- $\mathrm{d}=20 \mu \mathrm{m} ; \mathrm{e}-\mathrm{h}=50$ $\mu \mathrm{m}$.) 

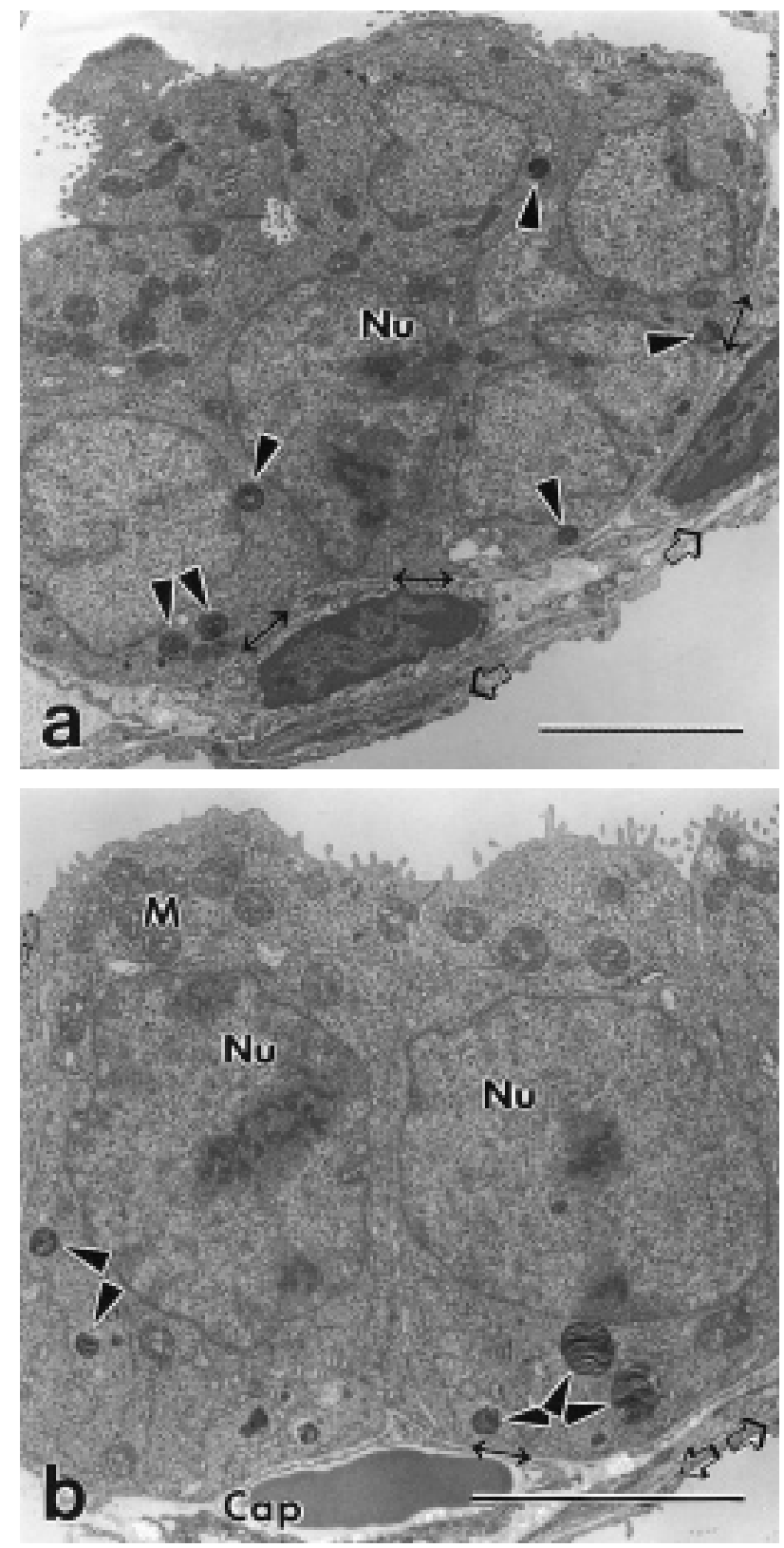

Fig. 5. - Transmission electron microscopy of experimental rat lung 2 days after intrabronchial instillation of rhKGF showing: a) an epithelial micropapilla composed of alveolar epithelial type II cells (AEII) containing lamellar bodies (arrowheads); and b) a region with AEII monolayer. Note that AEII are sitting on a basal lamina (double-headed arrows) and did not grow over any type I cell leaflet (open arrows). Cap: capillary; M: mitochondrion; $\mathrm{Nu}$ : nucleus. (Internal scale bars $=5 \mu \mathrm{m}$.)

successive cycles of mitosis after instillation of a single bolus of rhKGF.

Hyperplasia of AEII was also associated with changes in the immunohistochemical expression pattern of CD44v6. This integral membrane glycoprotein, which belongs to the group of cell adhesion molecules, is thought to be intimately involved in cell/cell and cell/matrix interactions in tumour development [33] and pulmonary fibrosis [31]. The shift of CD44v6 from basolateral towards lateral localization in AEII following rhKGF instillation was associated with the transition from epithelial micropapillae to cuboid monolayers and the spreading of intermediate

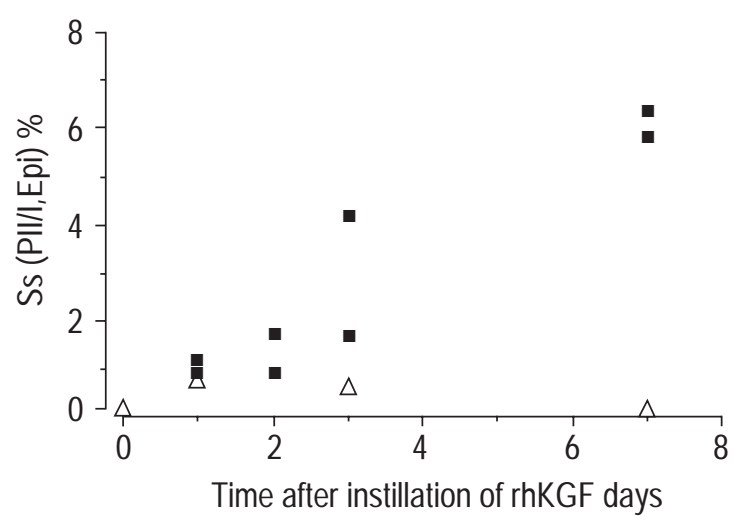

Fig. 6. - Increase in intermediate alveolar epithelial type II/type I cells (AEI) following intrabronchial instillation of recombinant human keratinocyte growth factor (rhKGF) versus into rat lungs estimated by means of stereology based on morphology and surfactant protein D immunohistochemistry. The increase in intermediate AEII/AEI cell coverage of the alveolar wall (Ss (PII/I,Epi)) day 0-7 following treatment with rhKGF $(\boldsymbol{\square})$ versus diluent $(\triangle)$ was statistically significant at a level of $\mathrm{p}=0.036$ tested by analysis of variation and partial correlation, respectively.

AEII/AEI. The prominent expression of CD44v6 protein and the lateral shift might indicate that the hyperplastic AEII have turned from a resting state into a state of migration, which would be necessary to spread over the alveolar surface when transforming into AEI. CD44v6 has been proposed to be causally involved in lung metastasis formation in a rat metastasis model [34], mediate chemotaxis, aggregation or attachment, depending on interaction with the respective ligand [35], and be important in migration during repair or neoplasia $[33,36]$. In pulmonary fibrosis, which is characterized by hyperplasia of AEII and which may result in a metaplastic cuboid epithelial lining of the airspaces [37], reduced CD44v6 staining was reported in several experimental models [19] and in fibrotic human lungs [38]. This may reflect a reduced ability of the hyperplastic AEII to migrate, which may favour the persistence of a cuboid epithelium rather than leading to spreading and differentiation into normal AEI.

Looking in detail into the alveolar epithelial cell phenotypes by means of immunohistochemistry and double immunofluorescence microscopy using well-established markers $[18,22,28]$, the present study revealed that the parenchymal architecture, at day 7 after instillation of rhKGF, was still different from untreated lungs or rat lungs instilled with saline. Although some of the cuboid hyperplastic AEII already exhibited faint apical staining for the AEI marker LEL at day 3 after treatment, there was a considerable increase, again quantified by means of stereology, in intermediate alveolar epithelial cells characterized by AEI shape as well as staining for the AEI marker LEL and by staining for the AEII markers SP-D and CD44v6. The high incidence of AEII/AEI intermediates indicates that a considerable proportion of hyperplastic AEII differentiated into AEI in vivo within 1 week following a single instillation of rhKGF. A shift towards AEI differentiation was also indicated by the increased staining intensity of AEII cells for cytokeratin 18, which was reported to be most prominent during postnatal proliferation and cytodifferentiation of alveolar epithelial cells [39] as well as during flattening and transformation of isolated 

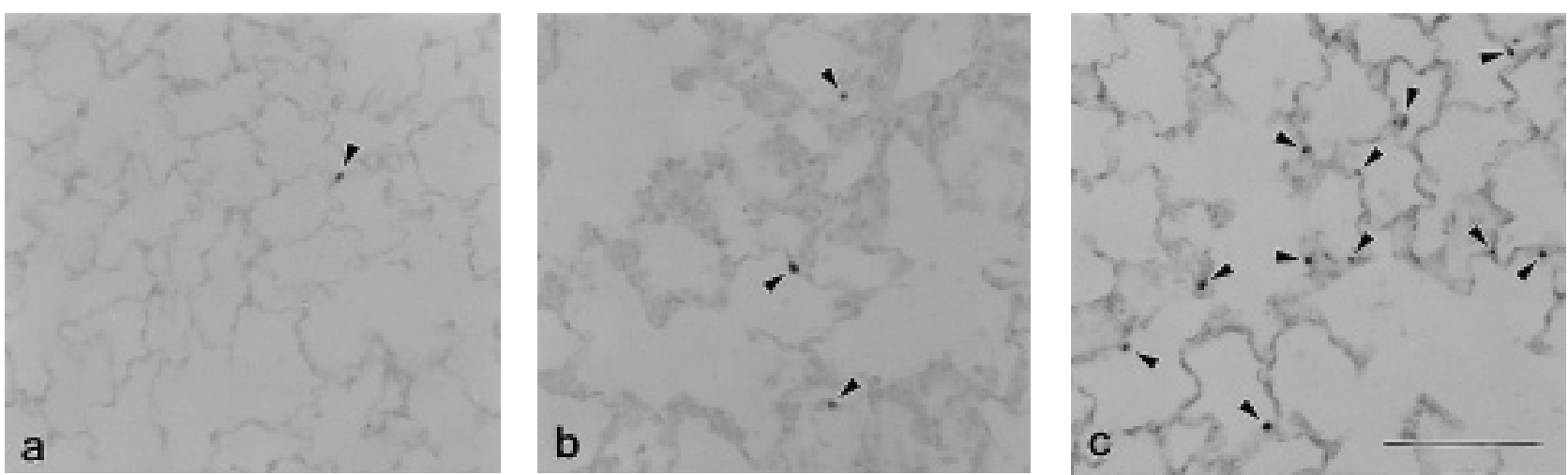

Fig. 7. - Terminal deoxynucleotidyl transferase-mediated deoxyuridine triphosphate nick end-labelling (TUNEL) of: a) control lung, 7 days after instillation of diluent; and b, c) lungs treated with recombinant human keratinocyte growth factor and fixed: b) 2 days; and c) 7 days after intrabronchial instillation. Note the highly increased incidence of TUNEL positive alveolar epithelial cells (arrowheads) at day 7 . (Internal scale bar=100 $\mu \mathrm{m}$.)

AEII in primary culture [40]. In vitro studies of isolated AEII investigating the effects of KGF on the expression of AEI-specific aquaporin-5 versus AEII-specific surfactant apoproteins (SP-A, -B, and -C) showed that the continuous presence of KGF may both prevent and reverse the progression towards an AEI phenotype [41]. Likewise, in embryonic lung development, continuous supplementation with KGF either by construction of transgenic SP-C/ KGF mice [42] or by culturing lung explants in the presence of exogenous KGF [43] resulted in the disruption of lung morphogenesis with the distal epithelium retaining AEII features.

While a considerable portion of the AEII differentiated into AEI, resolution of the "metaplastic" cuboid alveolar epithelium seen at day 3 after instillation of rhKGF was also accomplished by apoptosis as evidenced by the increased number of TUNEL-positive cells. It might be argued that this increase in apoptotic events may be a mere consequence of the increased number of AEII. However, since little is known about the time-frame detected by the TUNEL method, the apoptotic events detected may relate to a much higher frequency of cell death if only a narrow frame is seen by TUNEL. In analogy to the present findings, apoptosis of AEII has been proposed to be involved in the formation of the alveolar septa associated with the structural maturation of normal rat lungs during early postnatal development [29]. Extensive apoptosis of AEII has been observed in patients in the resolution phase of acute lung injury, and has been suggested to be largely responsible for the disappearance of AEII hyperplasia [44].

In the adult lung, proliferation of AEII is a common characteristic of the lung's response to injury. Evidence of AEII proliferation has been observed in lungs of patients in the recovery phase of adult respiratory distress syndrome, and most cases of interstitial lung diseases and pulmonary fibrosis, as well as in animal models of acute lung injury and pulmonary fibrosis [31, 37]. AEII proliferation has been linked to repair processes during the early phase of acute lung injury. Experimental studies have demonstrated that rhKGF can protect the lung against various forms of injury including acid instillation, $\alpha$-naphthylthiourea, hyperoxia, bleomycin and $\gamma$-irradiation [9-14]. In a recent clinical study, endogenous bioactive KGF was detected in the pulmonary oedema fluid from patients early in the course of acute lung injury [45], and preliminary data indicate that in mechanically ventilated patients only those suffering from adult respiratory distress syndrome

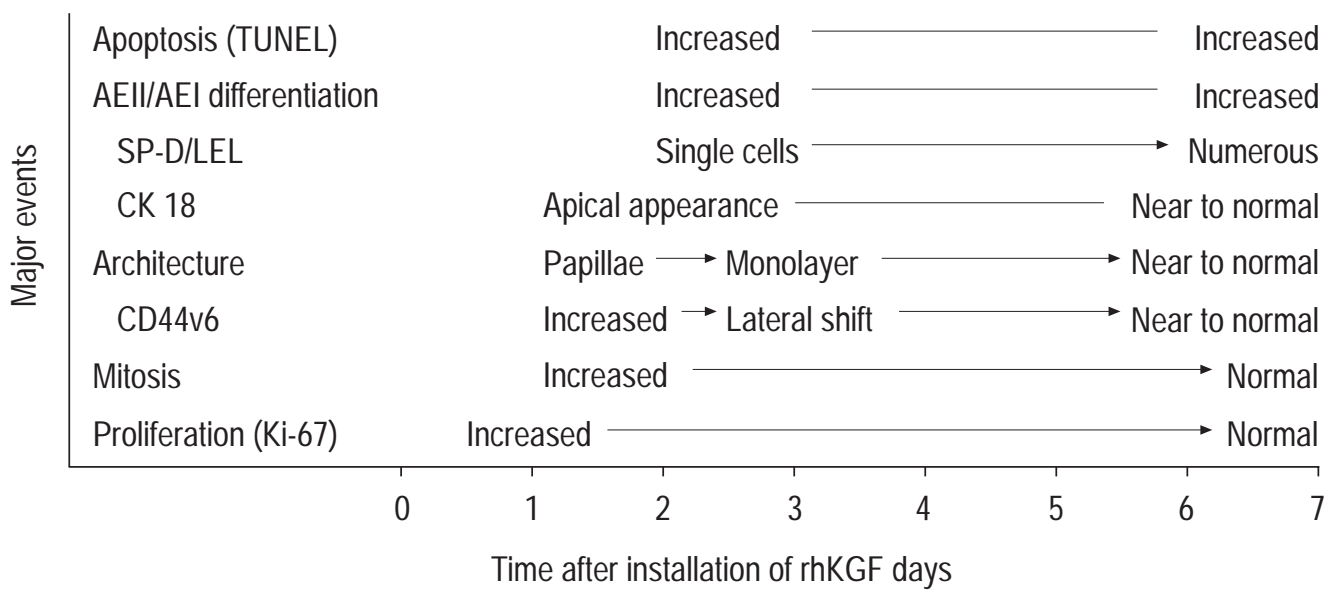

Fig. 8. - Schematic diagram summarizing the major events observed after intrabronchial instillation of recombinant human keratinocyte growth factor in rat lungs. The descriptions denote the time points of the first signs of the corresponding effects and the endpoints after 7 days of observation, respectively. Solid lines denote persistence of an effect, and broken lines indicate time points that were not examined. TUNEL: terminal deoxynucleotodyl transferasemediated deoxyuridine triphosphate nick end-labelling; AEII: alveolar epithelial type II cells; AEI: type I cells; SP-D: surfactant protein D; LEL: Lycopersicon esculentum lectin; CK 18: cytokeratin 18; CD44v6: the exon 6 containing isoform of CD44; Ki-67; a proliferation marker. 
had detectable amounts of KGF in their bronchoalveolar lavage fluid [46].

In conclusion, the results of the present in vivo study support the concept that keratinocyte growth factor not only stimulates proliferation and migration of epithelial cells but also stimulates cell differentiation [4, 45]. It has been demonstrated that restoration of a normal alveolar septum after experimental intrabronchial instillation of exogenous recombinant human keratinocyte growth factor is accomplished by both terminal differentiation and apoptosis of hyperplastic alveolar epithelial type II cells. Together with recent clinical data $[45,46]$, the findings lend further support to a role of keratinocyte growth factor in the reconstitution of the alveolar epithelial barrier both in experimental and clinical situations.

\begin{abstract}
Acknowledgements. Recombinant human keratinocyte growth factor was a generous gift from $\mathrm{T}$. Ulich (Amgen Inc., Thousand Oaks, CA, USA). The expert technical assistance of R. Petzold, I. Peterson and S. Langer in immunohistochemical and immunofluorescence staining, of M. Schoch in TUNEL staining, of B. Kettner in electron microscopy and of H. Seidel (all from the Technical University of Dresden, Germany) for photographic reproduction is acknowledged with thanks. The authors thank V. Dimmer (Technical University of Dresden) who performed the statistical analysis.
\end{abstract}

\section{References}

1. Finch PW, Rubin JS, Miki T, Ron D, Aaronson SA Human KGF is FGF-related with properties of a paracrine effector of epithelial cell growth. Science 1989; 245: 752 755.

2. Aaronson SA, Bottaro DP, Miki T, et al. Keratinocyte growth factor. A fibroblast growth factor family member with unusual target cell specificity. Ann N Y Acad Sci 1991; 638: 62-77.

3. Rubin JS, Bottaro DP, Chedid M, et al. Keratinocyte growth factor. Cell Biol Int 1995; 19: 399-411.

4. Werner S. Keratinocyte growth factor: a unique player in epithelial repair processes. Cytokine Growth Factor Rev 1998; 9: 153-165.

5. Panos RJ, Rubin JS, Csaky KG, Aaronson SA, Mason RJ. Keratinocyte growth factor and hepatocyte growth factor/ scatter factor are heparin-binding growth factors for alveolar type II cells in fibroblast-conditioned medium. $J$ Clin Invest 1993; 92: 969-977.

6. Adamson IY, Young L, Bakowska J. Enhanced alveolar type II cell growth on a pulmonary extracellular matrix over fibroblasts. Am J Physiol 1997; 272: L413-L417.

7. Sugahara K, Rubin JS, Mason RJ, Aronsen EL, Shannon JM. Keratinocyte growth factor increases mRNAs for SPA and SP-B in adult rat alveolar type II cells in culture. Am J Physiol 1995; 269: L344-L350.

8. Ulich TR, Yi ES, Longmuir K, et al. Keratinocyte growth factor is a growth factor for type II pneumocytes in vivo. $J$ Clin Invest 1994; 93: 562-572.

9. Guo J, Yi ES, Havill AM, et al. Intravenous keratinocyte growth factor protects against experimental pulmonary injury. Am J Physiol 1998; 275: L800-L805.

10. Yano T, Deterding RR, Simonet WS, Shannon JM, Mason RJ. Keratinocyte growth factor reduces lung damage due to acid instillation in rats. Am J Respir Cell Mol Biol 1996; 15: 433-442.
11. Mason CM, Guery BP, Summer WR, Nelson S. Keratinocyte growth factor attenuates lung leak induced by alpha-naphthylthiourea in rats. Crit Care Med 1996; 24: 925-931.

12. Yi ES, Williams ST, Lee $\mathrm{H}$, et al. Keratinocyte growth factor ameliorates radiation- and bleomycin-induced lung injury and mortality. Am J Pathol 1996; 149: 1963-1970.

13. Deterding RR, Havill AM, Yano T, et al. Prevention of bleomycin-induced lung injury in rats by keratinocyte growth factor. Proc Assoc Am Physicians 1997; 109: 254-268.

14. Yi ES, Salgado M, Williams S, et al. Keratinocyte growth factor decreases pulmonary edema, transforming growth factor-beta and platelet-derived growth factor-BB expression, and alveolar type II cell loss in bleomycin-induced lung injury. Inflammation 1998; 22: 315-325.

15. Kalina M, Mason RJ, Shannon JM. Surfactant protein C is expressed in alveolar type II cells but not in Clara cells of rat lung. Am J Respir Cell Mol Biol 1992; 6: 594-600.

16. Kasper M, Rudolf T, Hahn R, Peterson I, Müller M. Immuno- and lectin histochemistry of epithelial subtypes and their changes in a radiation-induced lung fibrosis model of the mini pig. Histochemistry 1993; 100: 367-377.

17. Kasper M, Albrecht S, Grossmann H, Grosser M, Schuh $\mathrm{D}$, Müller M. Monoclonal antibodies to surfactant protein D: evaluation of immunoreactivity in normal rat lung and in a radiation-induced fibrosis model. Exp Lung Res 1995; 21: 577-588.

18. Kasper M, Singh G. Epithelial lung cell marker: current tools for cell typing. Histol Histopathol 1995; 10: 155169.

19. Kasper M, Bierhaus A, Whyte A, Binns RM, Schuh D, Müller M. Expression of CD44 isoforms during bleomycin-or radiation-induced pulmonary fibrosis in rats and mini-pigs. Histochem Cell Biol 1996; 105: 221-230.

20. Kasper M, Reimann T, Hempel U, et al. Loss of caveolin expression in type I pneumocytes as an indicator of subcellular alterations during lung fibrogenesis. Histochem Cell Biol 1998; 109: 41-48.

21. Gerlach C, Sakkab DY, Scholzen T, Dassler R, Alison MR, Gerdes J. Ki-67 expression during rat liver regeneration after partial hepatectomy. Hepatology 1997; 26: 573-578.

22. Koslowski R, Dobbs LG, Wenzel KW, Schuh D, Müller M, Kasper M. Loss of immunoreactivity for RTI40, a type I cell-specific protein in the alveolar epithelium of rat lungs with bleomycin-induced fibrosis. Eur Respir $J$ 1998; 12: 1397-1403.

23. Fehrenbach H, Ochs M. Studying lung ultrastructure. In: Uhlig S, Taylor AE, Eds. Methods in Pulmonary Research. Basel, Birkhauser Verlag, 1998; 429-454.

24. Nomoto Y, Kuwano K, Hagimoto N, Kunitake R, Kawasaki M, Hara N. Apoptosis and Fas/Fas ligand mRNA expression in acute immune complex alveolitis in mice. Eur Respir J 1997; 10: 2351-2359.

25. Howard CV, Reed MG. Unbiased stereology. Threedimensional measurement in microscopy. Oxford, BIOS Scientific Publishers, 1998.

26. Rubin JS, Osada H, Finch PW, Taylor WG, Rudikoff S, Aaronson SA. Purification and characterization of a newly identified growth factor specific for epithelial cells. Proc Natl Acad Sci USA 1989; 86: 802-806.

27. Kasper M, Rudolf T, Verhofstad AA, Schuh D, Müller M. Heterogeneity in the immunolocalization of cytokeratinspecific monoclonal antibodies in the rat lung: evaluation of three different alveolar epithelial cell types. Histochemistry 1993; 100: 65-71. 
28. Bankston PW, Porter GA, Milici AJ, Palade GE. Differential and specific labeling of epithelial and vascular endothelial cells of the rat lung by Lycopersicon esculentum and Griffonia simplicifolia I lectins. Eur J Cell Biol 1991; 54: 187-195.

29. Schittny JC, Djonov V, Fine A, Burri PH. Programmed cell death contributes to postnatal lung development. $\mathrm{Am}$ J Respir Cell Mol Biol 1998; 18: 786-793.

30. Pinkerton KE, Gehr P, Crapo JD. Architecture and cellular composition of the air-blood barrier. In: Parent RA, Ed. Comparative Biology of the Normal Lung. Boca Raton, CRC Press, 1992; 121-128.

31. Kasper M, Haroske G. Alterations in the alveolar epithelium after injury leading to pulmonary fibrosis. Histol Histopathol 1996; 11: 463-483.

32. Yu CC, Woods AL, Levison DA. The assessment of cellular proliferation by immunohistochemistry: a review of currently available methods and their applications. Histochem J 1992; 24: 121-131.

33. Fasano M, Sabatini MT, Wieczorek R, Sidhu G, Goswami S, Jagirdar J. CD44 and its v6 spliced variant in lung tumors: a role in histogenesis. Cancer 1997; 80: 34-41.

34. Günthert U, Hofmann M, Rudy W, et al. A new variant of glycoprotein CD44 confers metastatic potential to rat carcinoma cells. Cell 1991; 65: 13-24.

35. Weber GF, Ashkar S, Cantor H. Interaction between CD44 and osteopontin as a potential basis for metastasis formation. Proc Assoc Am Physicians 1997; 109: 1-9.

36. Svee K, White J, Vaillant $\mathrm{P}$, et al. Acute lung injury fibroblast migration and invasion of a fibrin matrix is mediated by CD44. J Clin Invest 1996; 98: 1713-1727.

37. Crouch E. Pathobiology of pulmonary fibrosis. Am J Physiol 1990; 259: L159-L184.

38. Kasper M, Günthert U, Dall P, et al. Distinct expression patterns of CD44 isoforms during human lung develop- ment and in pulmonary fibrosis. Am J Respir Cell Mol Biol 1995; 13: 648-656.

39. Woodcock-Mitchell J, Mitchell JJ, Reynolds SE, Leslie $\mathrm{KO}$, Low RB. Alveolar epithelial cell keratin expression during lung development. Am J Respir Cell Mol Biol 1990; 2: 503-514.

40. Paine R, Ben-Ze'ev A, Farmer SR, Brody JS. The pattern of cytokeratin synthesis is a marker of type 2 cell differentiation in adult and maturing fetal lung alveolar cells. Dev Biol 1988; 129: 505-515.

41. Borok Z, Lubman RL, Danto Sl, et al. Keratinocyte growth factor modulates alveolar epithelial cell phenotype in vitro: expression of aquaporin 5. Am J Respir Cell Mol Biol 1998; 18: 554-561.

42. Simonet WS, DeRose ML, Bucay N, et al. Pulmonary malformation in transgenic mice expressing human keratinocyte growth factor in the lung. Proc Natl Acad Sci USA 1995; 92: 12461-12465.

43. Shiratori M, Oshika E, Ung LP, et al. Keratinocyte growth factor and embryonic rat lung morphogenesis. $A m J$ Respir Cell Mol Biol 1996; 15: 328-338.

44. Bardales RH, Xie SS, Schaefer RF, Hsu SM. Apoptosis is a major pathway responsible for the resolution of type II pneumocytes in acute lung injury. Am J Pathol 1996; 149: 845-852.

45. Verghese GM, McCormick-Shannon K, Mason RJ, Matthay MA. Hepatocyte growth factor and keratinocyte growth factor in the pulmonary edema fluid of patients with acute lung injury. Biologic and clinical significance. Am J Respir Crit Care Med 1998; 158: 386-394.

46. Stern J-B, Paugam C, Fierobe L, et al. Presence of keratinocyte growth factor (KGF) and hepatocyte growth factor (HGF) in BAL fluid of mechanically ventilated patients with or without ARDS. Am J Respir Crit Care Med 1999; 159: A694 [abstract]. 